Western University

Scholarship@Western

Aboriginal Policy Research Consortium International (APRCi)

2010

\title{
Children with FASD- Related Disabilities Receiving Services from Child Welfare Agencies in Manitoba
}

Don Fuchs

Linda Burnside

Sheila Marchenski

Andria Mudry

Follow this and additional works at: https://ir.lib.uwo.ca/aprci

Part of the Maternal and Child Health Commons, and the Substance Abuse and Addiction Commons

Citation of this paper:

Fuchs, Don; Burnside, Linda; Marchenski, Sheila; and Mudry, Andria, "Children with FASD- Related Disabilities Receiving Services from Child Welfare Agencies in Manitoba" (2010). Aboriginal Policy Research Consortium International (APRCi). 251.

https://ir.lib.uwo.ca/aprci/251 


\title{
Children with FASD- Related Disabilities Receiving Services from Child Welfare Agencies in Manitoba
}

\author{
Don Fuchs • Linda Burnside • Sheila Marchenski • \\ Andria Mudry
}

Published online: 8 January 2010

C Springer Science+Business Media, LLC 2009

\begin{abstract}
Fetal Alcohol Spectrum Disorder (FASD) is a serious social and health problem for the child welfare, health and education systems in North America and other parts of the world. This article describes the population of children in care of the child welfare system in Manitoba. Also this article will highlight the relevance of these research findings to aboriginal populations in Canada and its implications for international aboriginal/ indigenous groups. Finally, the implications for policy, practice are discussed and the article puts forward some directions for further research.
\end{abstract}

Keywords FASD $\cdot$ Disability $\cdot$ Children in care $\cdot$ Child welfare $\cdot$ Prevention

Fetal Alcohol Spectrum Disorder (FASD) is a serious social and health problem for the child welfare, health and education systems in North America and other parts of the world. Aboriginal children are significantly overrepresented in the number of children in care of child welfare authorities in Canada and this overrepresentation is even greater for Aboriginal Children with FASD (Fuchs et al. 2005). The term FASD describes a wide range of disorders caused by women drinking alcohol during pregnancy. These include Fetal Alcohol Syndrome (FAS), Partial FAS, Fetal Alcohol Effects (FAE), Alcohol-Related Neurodevelopmental Disorder (ARND) and Alcohol-Related Birth Defect (ARBD). Characteristics of FASD include growth deficiency, evidence of central nervous system neurodevelopmental abnormalities that result in intellectual and developmental delay, and facial dysmorphology (changes in the shape of the face). Children with alcohol-related disorders often have cognitive and behavioural difficulties that cause them to have problems in school and society.

This article describes the population of children in care of the child welfare system in Manitoba and reports on the results of a study that was aimed at gathering data on the trajectories of children with FASD in care, and compares those trajectories to the trajectories of children with other disabilities and children with no disabilities. In addition, to illustrate the magnitude of concern for the risk of continued growth in the numbers of

D. Fuchs $(\bowtie) \cdot$ L. Burnside $\cdot$ S. Marchenski $\cdot$ A. Mudry

University of Manitoba, Winnipeg, MB, Canada

e-mail: fuchs@cc.umanitoba.ca 
children with FASD coming into care, the article reports the results of a study of women of child bearing age receiving service from Addictions Foundation of Manitoba (AFM). Also this article will highlight the relevance of these research findings to aboriginal populations in Canada and its implications for international aboriginal/indigenous groups. Finally, the implications for policy, practice are discussed and the article puts forward some directions for further research.

\section{FASD as a Significant Addiction and Mental Health Concern}

Fuchs et al.'s (2007) estimations of the costs of supporting FASD children in the child welfare system is the first of its kind in Canada. As identified by Hutson (2006) in her review of the cost of substance abuse in Canada, medical and education costs are better understood, particularly in the U.S. However, the author noted a lack of research in two areas that are critical to aggregating accurate costs incurred by the FASD population: the child welfare system and the justice system. Recently, the National Center on Addiction and Substance Abuse at Columbia University (2009) found that the second largest share of federal public spending for children in the U.S. includes the child welfare system for foster care, independent living and other related programs. These expenditures fall within a larger budget category, referred to as Child and Family Assistance. In fact, child welfare accounts for $3.1 \%$ of the total federal budget and $6.2 \%$ of state budgets spent on substance abuse and addiction.

Hutson (2006) has asserted that an estimated " $50 \%$ of children in care in Alberta, Canada have FAS" and she concluded that FASD-affected children may very well be overrepresented within the child welfare system (Hutson 2006). In Manitoba, Fuchs et al. (2005) found evidence of diagnosed or suspected FASD in $17 \%$ of the children in care in this province. Consequently, gathering accurate costs incurred on behalf of alcohol-affected children in the child welfare system is a critical phase in economic costing research around FASD.

More recently, health analysts in Alberta have estimated two types of aggregated costs in 2008 dollars. The first calculation is the aggregated lifetime costs for FASD-affected children multiplied by the number of affected children born each year. Based on their methodology, the annual long-term costs of FASD range from $\$ 130$ to $\$ 400$ million per year in Alberta. In a second calculation, the analysts used an FASD cost calculator developed by colleagues in North Dakota in order to estimate the annual short-term economic cost, which ranged from $\$ 48$ to $\$ 143$ million per year in 2008 Canadian dollars. This calculation estimates the annual costs incurred by people who are currently affected by FASD. Though these are rough estimates at best, the study effectively affirms the urgent need for primary prevention strategies ${ }^{1}$ in this country (Thanh and Jonson 2009).

It should be noted that there are currently no accurate national statistics on the rates of FASD in Canada. However, the incidence has been estimated at 1-6 in 1,000 live births (Stade et al. 2006), and women give birth to approximately 3,000-4,000 FASD-affected babies each year in Canada (Hutson 2006; Thanh and Jonson 2009). Moreover, the incidence of FAS is estimated at 1 case in 100 live births, which is thought to be higher than the U.S. (Stade et al. 2006; Lupton et al. 2004). Two Manitoba studies are noted. Square

\footnotetext{
${ }^{1}$ Primary prevention costs are more preventive-focused. One example includes public awareness campaigns preventing prenatal alcohol use in high-risk populations, since FASD is known to be preventable.
} 
(1997) estimated the prevalence in a Manitoba First Nations community to be 55-101 per 1,000 and Williams et al. (1999) reported an incidence of 7.2 per 1,000 live births in northeastern Manitoba. In considering these estimates, it is important to remember that the complexity of diagnosis contributes to the delay or even omission of diagnosis (Chudley et al. 2005), and prevalence is often determined by diagnostic thresholds (Klug and Burd 2003).

\section{Profile of Children with FASD in Care of the Child Welfare in Manitoba}

Fuchs et al. (2005) investigated the characteristics of 1,869 children with disabilities who were receiving child welfare services in Manitoba on September 1, 2004. Their initial study found that many of these children were described as having FASD. Consequently, this study provided a unique opportunity to report the characteristics of children with FASD within the population of children with disabilities in the provincial child welfare system.

A definition for "children with disabilities" was developed in accordance with the World Health Organization's perspective on disability, which highlights both personal and environmental factors (WHO 2003). In this study, children with disabilities are those whose ability to participate in age-appropriate activities of daily living was compromised by limitations in one or more areas of functioning. These children, according to this definition, also require supports to meet their unique needs. Included were children with congenital conditions, complex medical needs, chronic psychological or mental health concerns, and/or learning difficulties, as well as those with FASD. Six main types of disabilities were examined: intellectual, mental health, medical, physical, sensory and learning. FASD is usually considered to be an intellectual disability, but is also described as developmental delay in children, or as cognitive impairment.

The most frequently reported types of disabilities identified in the study were intellectual and mental health disabilities. Thirty-three percent of Manitoba's children in care had a disability and, of this group, $75 \%$ had an intellectual disability and $56 \%$ were diagnosed with, or suspected to have, a mental health related disability. Multiple disabilities were common, with the most frequently co-occurring types being intellectual and mental health disabilities.

FASD was diagnosed in approximately $34 \%$ of children in care with disabilities, or $11 \%$ of all children in care in Manitoba. Of the 1,403 children in care with an intellectual disability, $46 \%$ had diagnosed FASD. The proportion of boys to girls among those diagnosed with FASD (61\% boys and 39\% girls) was very close to the $60: 40$ male:female ratio found in the general disability population of children in care in Manitoba. It is also consistent with the overrepresentation of males within the intellectual disability population. The average age of the children with FASD was 10.08 years. They were, as a group, slightly younger than the general disability population of children in care, who had an average age of 10.75 years.

The overwhelming majority of children with FASD (89\%) were in the permanent care of a child welfare agency. In comparison, $61 \%$ of the general population of children with disabilities in care were in permanent care. A high percentage of the children with FASD $(86.9 \%)$ came into care as permanent wards. The reasons for children with FASD coming into care were predominately related to a difficult parental situation $(62 \%$ of children came into care because of the conduct of the parents or the incapacity of the parents to fulfill their parental role). Only $6 \%$ of the children with FASD came into care because of reasons that were related to the child's condition or the child's conduct. By comparison, in the non- 
FASD population, $54 \%$ of children were in care for reasons related to parental care and $18 \%$ were in care for reasons related to child's conduct or conditions. Many children with FASD had co-occurring disabilities. Mental health disabilities occurred in $46 \%$, while $20 \%$ had a medical condition, $17 \%$ had physical impairments, $3 \%$ had a learning disability, and $2 \%$ had a sensory disability. In total, $88 \%$ of children with FASD had co-occurring disabilities.

\section{Relevance to Aboriginal Populations in Canada}

\section{Aboriginal Children with FASD Overrepresented in Care}

Although U.S. studies suggest that FASD may be more prevalent in disadvantaged or impoverished populations, little is known about the prevalence of FAS-related disorders in Canadian child welfare systems, or how the unique needs of children with this disability are being met when they are in care.

Like some other jurisdictions in Canada (First Nations Child and Family Caring Society 2005; Trocmé et al. 2005), Manitoba's children in care population reflects an overrepresentation of Aboriginal children. The reasons for this over-representation have been acknowledged to stem from historical events, most significantly colonization, residential schools, and the subsequent intervention by the mainstream child welfare system (McKenzie and Morrissette 2003), resulting in disconnections from language, culture, and family. Coping with these disconnections has been challenging at best, and many struggle in their lives with alcohol dependence, violence, and other self-abusive behaviours. Research suggests that many First Nations children are removed from their families as a result of neglect (Blackstock et al. 2004; Trocmé et al. 2005). Neglect is considered to stem from poverty and poor housing, conditions which are endemic in the Aboriginal community and often beyond the control of individual families to change, and also from substance misuse (Trocmé et al. 2004). Further, the resources and services to assist Aboriginal families and their communities to address the issues of poverty, poor housing, and substance misuse are often inadequate (Trocmé et al. 2004, First Nations Child and Family Caring Society of Canada 2005).

The report of the Standing Committee on Human Resources Development and the Status of Persons with Disabilities (2003) found that the rate of disability is twice as high for Aboriginal Canadians compared to the non-Aboriginal population. For many Aboriginal children with disabilities, the required services related to their special care needs are often not available in their home communities, resulting in many families turning to the child welfare system to access supports and services. Additionally, the report indicates that the rates of Fetal Alcohol Spectrum Disorder (FASD) in some Aboriginal communities may be significantly higher than in non-Aboriginal populations. As a result of the lack of services for Aboriginal children with disabilities and the factors related to neglect and the incidence of FASD, there is an increased risk of Aboriginal children with disabilities coming into care.

This study found that $81 \%$ of the children with FASD in care in Manitoba were First Nations (Treaty Indian). The cultural origins of the remainder of the children in care with FASD were Métis (9\%), non-Aboriginal (5\%), non-status Aboriginal (4\%), or not known (1\%) (Fuchs et al. 2005).

The rate of disabilities for Aboriginal people has been reported to be twice as high as that of the non-Aboriginal population in Canada, and the rate of FASD in some 
Aboriginal communities may be significantly higher as well. In addition, First Nations families who live on reserves often may be disadvantaged in receiving services for their children with disabilities, since reserves fall under federal jurisdiction and social services are typically provided by provinces and territories. For these reasons, there may be an increased risk of Aboriginal children with disabilities coming into care of child and family service agencies to receive necessary disability services.

\section{Services for Individuals Living on Reserve}

Many of the children in care in Manitoba have First Nations status and agencies provide care on and off reserve. In Manitoba, parity in child welfare funding to support children on and off reserve has not been reached. Children on reserve remain disadvantaged in terms of services. This difference becomes even greater as children reach adulthood. Adult services, as described here, are not available for persons living on reserve. Individual Bands provide for their members as they are able and see fit. The extended families of many children in care live in First Nations Communities. The lack of on-reserve disability services may mean that children and youth in care and youth leaving care must choose between obtaining whatever services they can and living with the support of extended family. The right to reasonable services should be recognized for all residents of Manitoba.

In their study of children in care with disabilities in child and family services agencies in Manitoba, Fuchs et al. (2005) concluded that $17 \%$ of all children in care were affected by FASD. An overwhelming majority (89\%) of children with FASD was in permanent care of an agency and had limited contact with biological parents. In addition an overwhelming percentage (over 80\%) are of Aboriginal decent. Consequently, child welfare agencies in Manitoba have a significant care responsibility for these children until their age of majority, requiring agency staff and caregivers to be cognizant of the special needs of this population during childhood and adolescence and through the transition to adulthood. In addition, there is great need for culturally sensitive services delivered by culturally competent workers. There is a good deal of evidence to indicate that this is similar to other jurisdictions in Canada and the United States (Brown et al. 2006).

\section{Research Context and Objectives}

The study reported in this article is the second phase of the original research by Fuchs et al. (2005) identifying children in care with disabilities. It looks more closely at children with FASD and their history as they enter the child welfare system and come to rely on the parenthood of the state. It was conducted by the Faculty of Social Work, University of Manitoba and the Child Protection Branch of the Manitoba Department of Family Services and Housing under the auspices of the Prairie Child Welfare Consortium. Funding was provided by Health Canada with the support of the Centre of Excellence for Child Welfare.

In recognition of the high care needs and the over-representation of children with disabilities in the Manitoba child welfare system, as identified in the previous research of Fuchs et al. (2005), this study was aimed at developing a fuller understanding of the pathways into care for children with disabilities. In addition, the 2005 study found First Nations and Métis children were significantly over-represented in the population of children in care with disabilities. Because of the significant proportion of Manitoba children in care identified with a diagnosis of FASD, and because of the over-representation of First Nations 
Children, understanding the relationship between this population and child welfare agencies was particularly important. Information on the evolution of child welfare interventions from an initial admission into care resulting in a first legal status, to subsequent legal statuses including the possibility of a permanent order of guardianship, has implications critical to prevention, intervention, and permanency planning for children with disabilities. Information on the placement and legal status histories of children and comparative analysis of children with and without disabilities is greatly needed at all levels of the service delivery system, from practitioners to policy-makers, for enhancing capacity to meet the needs of children with disabilities.

\section{Design and Methodology}

This project sought to examine the legal status and placement histories of children with disabilities and compare this to the histories of children without a recorded disability in the care of mandated child protection agencies in Manitoba as of December 1, 2005. The researchers were able to use as a study population a cohort of children in care with and without disabilities who were identified in their previous research and who were still in care (Fuchs et al. 2005). This helped ensure that there were two discrete groups for comparison purposes. It should be noted here that based on the findings of the earlier study, this study focussed intentionally on children with and without disability who were in permanent care of the child welfare agencies in the Province

\section{Comparing in Care Trajectories of Children with Disabilities and Children with No Disabilities}

For the purposes of this phase of the research project, only children who were permanent wards or were under Voluntary Placement AgreementsVPAs on December 1, 2005 were examined as part of the comparison groups. Although they were all either permanent wards or under a VPA on that date, their legal status histories could consist of a variety of legal statuses and may have included Apprehension, Temporary Ward, Permanent Ward (PW), Transition Planning, VPA, Voluntary Surrender of Guardianship (VSG) and/or Unknown legal statuses.

Children with permanent ward status were further examined to determine the length of time from their first legal status to the time they became permanent wards. The FASD PW population had the shortest period of time between the two legal statuses ( $\mathrm{Mdn}=$ 1.30 years). At just over 2 years on average between their first legal status and becoming a permanent ward, this was almost a year shorter than other children who became permanent wards. The No disability PW group was just over 3 years ( $M \mathrm{dn}=2.12$ years), as was the CWD PW no FASD group ( $M \mathrm{dn}=2.41$ years). This may be partially due to the legal status legislative requirements under the Child and Family Services Act (1985) that apply to children who are involved with the child welfare system at different ages, as discussed earlier. Since the PW FASD group were first involved with the system at Mdn age of 1.29 years of age, the amount of time they could be in care under temporary status is less than children who are older than 5 years of age when they first come into care.

Because the age of children was a factor that influences the length of their total time in care, a more accurate comparison of the relative time spent in care by the legal status disability groups could be made by comparing the children's time in care as a proportion of their ages. 
Although the legal status and placement status of children are linked, there may be differences between the amount of time children have a legal status and the amount of time they are actually in an agency supported placement. The history of a child with an agency may involve a variety of placement arrangements, and it was important to understand whether the placement history of children varied by their disability.

The mean length of time in placements of children in the six legal status by disability groups was determined and compared. As expected, permanent wards spent the longest time in placement. However, of the permanent wards, those with FASD spent the most time in child welfare agency placements $(M d n=7.42$ years, $)$, compared to permanent wards with other disabilities $(M d n=5.67$ years, and permanent wards with no disabilities $(M d n=$ 4.32 years).

The total length of time each child had spent in an agency placement was calculated as a proportion of their age at December 1, 2005. A mean of the proportions was determined for each disability legal status group. The comparison of mean proportions showed that children with FASD spent on average greater than $70 \%$ of their lives in care $(M \mathrm{dn}=76.52)$. This was a higher proportion than permanent wards with no disabilities $(M \mathrm{dn}=58.35 \%)$, and considerably higher than permanent wards with other disabilities $(M \mathrm{dn}=52.34 \%)$. This was a particularly important finding as it has major implications for policy, programs and training of social workers and foster care service providers which will be discussed in more detail later in this article.

It is clear from these findings that permanent wards who received a diagnosis of FASD have come into care for the first time at a younger age (2.5 years) than children with no disability (3.6 years) and at a considerably younger age than children with other disabilities (4.3 years). The data also demonstrated that children in the FASD group became permanent wards more quickly than children in either of the other permanent ward groups. Their time from first legal status to a permanent order was approximately 2 years, compared to 3 years for other children. This fast track, compounded by an earlier initial legal status, results in the children with FASD becoming permanent wards at a much earlier age, approximately 4 years of age, compared to 6 years of age for children with no disabilities and over 7 years of age for children with other disabilities.

Given the shorter time period between first legal status and the granting of permanent guardianship, one would expect that the FASD permanent ward group would have a lower mean number of legal status changes. ${ }^{2}$ That is precisely what the data demonstrated. Fewer opportunities were occurring for these children to be re-united with their families of origin. Once the children were in care, they moved comparatively quickly and directly to becoming permanent wards. As previously discussed, this shortened pathway into care for children with FASD may be entirely a consequence of the conditions imposed by the Child and Family Services Act (1985). What this research demonstrated was that the conditions specified in the Act appear to have a greater impact on the legal status history of children with FASD, as a result of their younger age at admission to care.

Finally both the legal and placement histories confirm that permanent wards with FASD were spending, on average, approximately three quarters of their lives in the care of an agency, about $15 \%$ more than any other children who are permanent wards.

\footnotetext{
${ }^{2}$ Although there are rare exceptions to this rule, it is generally true that once a child becomes a permanent ward, he/she will have no more legal statuses.
} 
In summary Children with FASD who become permanent wards in Manitoba tended to be of aboriginal ancestry and tended to become children in care of an agency at a much earlier age than other children with disabilities or children with no disabilities. Once in care, they were more likely than other children to continue in agency placements. They become permanent wards more quickly than other children.

\section{Women of Child-Bearing Age with Alcohol Problems in Manitoba and the Risk for Children with FASD Coming in Care}

After a thorough and systematic search of the literature, the researchers were able to determine that the there were no well developed studies of prospective cohorts in Canada have been used to establish the prevalence of FASD. Consequently as the researchers were able to access the following data from the population of women of child bearing age receiving treatment for alcohol abuse, it was used to give some indication of the magnitude of the concern of FASD in Manitoba. Further recognizing the significant number of alcohol-affected children in the child welfare system, the researchers felt it was important to learn more about the possible number of children in Manitoba who might be similarly affected, and about the women who might give birth to them. There were no reliable provincial statistics identifying the number of children with FASD in the general population, as there are in all probability many women using alcohol during pregnancy who are not known to the social services, medical, or legal professions. Still, to begin to develop an awareness of the scope of this issue, information on one particularly vulnerable group of women was obtained from the Addictions Foundation of Manitoba (AFM): women of child-bearing age (19-45 years) who were involved with an addictions program in 2005-2006, the year of the study. This sample is not intended to be representative of Manitoba women who might have children affected by FASD, but rather to serve as an example of one group, among many, of women who appear to be at risk for having such children.

AFM provided summary data on women who participated in screening for addictions related programs. The data reported here represents all of the women who participated in the screening process for whom alcohol abuse determined to be a problem. Many of the women who sought treatment were compelled to seek treatment, either because of a probation order or as a requirement for the return of their of children from care. Their screening tool is a self-report questionnaire completed by all potential AFM clients. AFM reported that in 2005-2006 the number of women in the 19-45 year age group who participated in screening was 1212 . The number of respondents varied from item to item in the screen with a range for general questions from a high of 1212 answering the question "How may children do you have?" to a low of 584 answering "Have you ever attempted suicide?" The average number of respondents to general questions was 1084. Although women might be involved with AFM for any addiction, over $90 \%$ reported feeling a need to cut down on alcohol or drug use.

Of particular interest to this study is the potential number of children in situations of maternal alcohol abuse. In this population, 65 women ( $6 \%$ of respondents) were pregnant at the time they filled out the screen. The number of women who responded to the question "How many children do you have?" was 1200. Of those, 312 reported no children, 230 had one child, 264 had two children, 207 had three children, 104 had four children, 70 had five children, and 52 had six or more children. Based on their report and using six as the maximum number of children, we can conclude that these mothers account for at least 
2,457 children. Although 312 women reported having no children, 669 women indicated that they had no children living with them.

Although there may be other explanations for children not living with their mothers, many of these mothers have, at least temporarily, lost custody of their child/ren to an agency. Child and Family Services (CFS) was noted as the referral source for 234 women. When asked about specific events that occurred in the past year, 370 women reported the loss or placement of a child in care of a CFS agency. It is not possible from the information provided to determine how many children were involved or whether there were previous apprehensions. However, the screening tool does gather information on current involvement with the legal system. Of 479 women who indicated being involved with the legal system, 156 reported a Child \& Family Services Order as the nature of that involvement. When asked if alcohol was related to their involvement in the court system, 291 women indicated that it was. In summary, there is evidence that approximately one third of women screened for acceptance into an AFM program have been involved with a child and family service agency.

It is possible from the data collected to describe the main socio-demographic characteristics of the group of women 19-45 years completing the AFM screening in 2005/06. The majority, approximately $70 \%$, were from the Winnipeg region, which includes the city of Winnipeg and extends as far west as Portage la Prairie and includes all of southeast Manitoba. Approximately half of the women (615 or 51\%) had not completed high school: 181 (15\%) had less than grade 9 education, and $434(36 \%)$ had incomplete high school. Of the remaining half, 244 (20\%) had completed high school, 203 (17\%) had some college or university, and $99(8 \%)$ had a college or university degree.

Only 309 (26\%) of these women were employed: 202 (17\%) employed full-time and 107 (9\%) part-time. Women most commonly described themselves as unemployed 578 (49\%). Another 119 (10\%) described themselves as homemakers. For those completing this item, household incomes were generally low with 365 (31.7\%) reporting an income of less than $\$ 10,000$ and $175(15 \%)$ citing the $\$ 10,00-\$ 19,000$ category. Only 244 (21\%) women reported household incomes above $\$ 20,000$ with 88 of those in the $\$ 20,000-\$ 29,000$ range. This item was poorly completed: 287 (25\%) responded "don't know" and another 80 (7\%) declined to answer.

Present marital status was single for 661 (57\%), married/common law for $283(25 \%)$, divorced/separated for 164 (14\%), and widowed for 10 (1\%). Half of the women (541) described themselves as having been seen at some time for emotional or mental health issues, and $594(53 \%)$ have at some time been prescribed antidepressant medications. Emotional or mental health issues were serious enough to have resulted in hospitalization for $231(22 \%)$. Violent behaviour when either sober or straight was reported by $329(29 \%)$ women and self-reports of violent behaviour increased to 620 $(55 \%)$ when using alcohol or other drugs. An overdose of drugs or alcohol was reported by $339(31 \%)$ women in the group and had occurred within the past 12 months to 157 women.

In summary in Manitoba in 2005-06, more than 1200 adult women of childbearing age were screened for services related to addictions at the Addictions Foundation of Manitoba, which is only one of the service providers for women of childbearing in Manitoba. These women tended to be single, unemployed, many with less than a high school education, of low economic status and half had a history of emotional or mental health issues. They were mothers to 2500 children but were not currently custodial parents to all of their children. In addition it is important to note there were 312 women who reported having no children. 


\section{Implications for Child and Family Service Policy and Service Provision}

The data from these studies would strongly indicate that social workers need to be aware of the possibility that children for whom they are providing service may be alcohol-affected. Workers need to know the characteristic physiology and behaviour patterns that are an indication of the condition, and pursue formal assessment for children they suspect may have FASD. In addition to understanding how this disability affects the child's functioning and service needs, workers need to be trained to recognize and help alleviate the additional stressors faced by families caring for a child with FASD. Because children with FASD come into care earlier and spend more of their life in placement, workers must recognize the even more critical role of permanency planning for them. There are some services available in the community for children with FASD and/or their families. Workers need to know about the availability of services in their region and be able to advocate for FASD related services for both children in care and children in danger of coming into care and their caregivers.

Similarly, expertise related to FASD is critical for foster parents and other direct service providers. They must be prepared to manage the unique needs of children with this condition. Recognizing the long-term placement needs of these children, foster parents need to be able to make a long-term commitment to their care. They must be aware of the additional stresses that may result from caring for children with FASD and develop some reliable stress management strategies. They also must learn to work with the children and youth to build cultural connections with the children and communities.

It is not enough to plan for the needs of children while they are in care. It is important to begin planning for children for life after care, beginning while they are still in care. Every year increasing numbers of children who have been identified with FASD will be transitioning out of care and into the community. The shift to independence is difficult for all children with disabilities. This is due in part to the significant differences in the structure of service delivery for children and adults. The move to independence for persons with FASD is further complicated by the nature of their disability. They are often not eligible for services related to cognitive impairments because their level of intellectual functioning is above the eligibility criteria. There are few if any adult services directly related to FASD. As adults, their disability tends to be invisible, but their behaviour can present many challenges. Long term planning for children with FASD needs to include special attention to their transition into adulthood and conceptualization and consideration of lifespan planning should begin in childhood.

In summary, workers, foster parents and other service providers must be prepared to provide the kind of care that best supports children presenting with this configuration of needs. Connections to FASD expertise must be made to adequately provide for the needs of alcohol-affected children. Whether this means increasing the expertise within CFS or integrating FASD services with other service providers, it is essential to have knowledge and skills related to FASD available to every affected child in care.

The review of the number of women of child-bearing age who are involved with AFM gives a cursory indication of the scope of addiction issues among women in the Province of Manitoba. The potential for children to be affected by alcohol both biologically as a result of prenatal exposure, and environmentally as a result of postnatal exposure, is significant. The child welfare system appears to be a primary intervener with this high risk population. 
CFS policy direction and resource allocation need to recognize the prevalence and complex needs of this group.

Beyond the scope of Child and Family Services, the AFM data emphasized the importance of prenatal alcohol prevention programs and the potential need for supports for women and families. It is also an indication of the continued importance of early childhood intervention and identification of children and families requiring support.

The potential exist in many jurisdictions to develop a service model across divisions that would encompass the provision of early childhood intervention and child care, family supports, vocational/employment strategies, independent living supports, and affordable housing. Integrated service delivery on this scale would make social inclusion of persons with FASD possible. An integrated approach would reduce the demand on the overly subscribed child welfare system in Manitoba and provide greater access to the range of appropriate services required by the children and families with FASD disabilities.

\section{Implications for Policy}

\section{Culturally Appropriate Intervention and Prevention Models}

The study clearly identified unmet needs for support services available to children with disabilities and their families. There may be many reasons why children are not receiving these services. It could be a result of problems in documenting needs or communication gaps between child welfare workers and children with disability workers, budget restraints or a lack of qualified support providers. The first two possibilities were previously discussed under implications for practice/service delivery. Additionally, support services may not be available in the child's community.

To ensure that adequate support services are in place for the growing number of children with complex and multiple disabilities, there will need to be additional resources allocated to ensure the availability of culturally appropriate support resources and the development of new training programs which promote cultural competence amongst agency workers and foster parent resources.

Culturally appropriate interventions and prevention models reflecting Manitoba's culturally diverse population are essential. This is particularly important in terms of First Nations people, who make up the largest percentage of children in care with a disability. Cultural effects may be further compounded by significant family problems such as poverty and domestic violence.

Effective prevention and intervention programs need to be developed that respect Aboriginal cultures and are structured in a way that will promote accessibility and participation. Such programs would need to address issues related to understanding disabilities and the additional stress placed on families caring for children with disabilities as well as resources available to families.

All the studies reported in this article found that substance abuse was a major factor in the origin of disability in many children. This suggests there is a particularly strong need for culturally appropriate FASD prevention programs and culturally relevant services for children with FASD related disabilities. 


\section{Conclusions and Directions Future Research}

Fetal Alcohol Spectrum Disorder is an area of significant concern in Manitoba's child welfare system, with $11 \%$ of all children in care having FASD. More than one third of the children in care with disabilities have been diagnosed with FASD. This is an entirely preventable condition, as it results from women drinking alcohol while pregnant. Its prevalence in the population of Aboriginal children with disabilities, especially First Nations children, indicates a strong need for establishing and expanding programs that emphasize the importance of not drinking any alcohol if a pregnancy is being planned, or, especially, during pregnancy. There is a strong need for such programs to be accessible and culturally appropriate.

A number of important questions arise from this research. They are related to preferred practices in service provision, policy and prevention. Although children in care with FASD have now been identified and their legal and placement histories described, they are still largely unknown. What are the reasons they come into care, what is the nature of their relationship with their family of origin, their siblings, and their extended family? What are the social problems experienced by the biological mother / father that contribute to the need for care? Are there opportunities for maintaining family relationships? What are needs of sibling groupings of children with FASD? What are the factors that make it possible for some placements to be sustained over long periods of time? What is their success in achieving independence? A longitudinal study of a cohort of children from families dealing with addictions would offer an opportunity to obtain information of immeasurable value.

The knowledge that children with FASD in Manitoba are spending such a large proportion of their lives in care makes it even more important to understand their needs and be able to meet them. Most children in care in Manitoba are in foster homes. What are the support needs of foster families who are fostering children with FASD? If children are in long term foster care, what is the best way to increase the stability of their placements?

As children with FASD enter adulthood, it is clear that they will need some continuing support. What is the most effective way to assist them in their transition out of agency care? What is happening in other jurisdictions?

As indicated earlier the findings reported in this article show that children with FASD come into care in Manitoba earlier and become permanent wards more quickly than other children. This raises the question of the efficacy of the permanency planning provision of the Provincial Child and Family Service legislation in relation to children with FASD. It appears that Legislation's intention to meet the needs of children for a stable permanent home have a particular impact on children with FASD because they enter the child welfare system in Manitoba at an earlier mean age than other children. If there was more time to support families struggling with addictions, would it be possible for more children with FASD to return to their families of origin? This issue may not be adequately addressed without a concurrent increase in community addiction services to better and more quickly support these families when children first come into care. In a related question, can voluntary placement agreements be used more effectively to maintain parental involvement in the care of children with FASD?

The data from AFM identifies a high risk population of women. What type of prevention programs would be most successful in addressing a high risk population? Although beyond the scope of the child and family services system, it would be of 
great importance to prevent children coming into care by preventing incidence of FASD.

Children in care of child welfare system present an additional challenge to the already over stretch child welfare systems that work indigenous peoples in many parts of the world. The data on the increasing number of children coming into care and the prevalence data from the Addictions Foundation Manitoba indicates that there is a growing degree of urgency for health, education and child welfare system to develop effective integrated health and service policy and programs to respond to needs of the increasing numbers of children and families with FASD and to develop broad cross sectoral preventive strategies. Truly, there is great need for much more immediate action to address this complex and compelling social issue.

\section{References}

Blackstock, S., Trocme, N., \& Bennet, M. (2004). Child welfare response to Aboriginal and Caucasian children in Canada: A comparative analysis. Violence against Women, 10(8), 901-916.

Brown, J., Bednar, L., \& Wiebe, B. (2004). Motives of currently licensed Manitoba foster parents. Envision: The Manitoba Journal of Child Welfare, 3(2), 1-13.

Chudley, A. E., Conry, J., Cook, J. L., Loock, C., Rosales, T., \& LeBlanc, N. (2005). Fetal alcohol spectrum disorder: Canadian guidelines for diagnosis. Canadian Medical Association Journal, 172, 1-21.

First Nations Child and Family Caring Society (2005). Wen; De Report, Ottawa: Ontario: FNCFCS.

Fuchs, D., Burnside, L., Marchenski, S., \& Mudry, A. (2005). Children with disabilities receiving services from child welfare agencies in Manitoba. Retrieved December 1, 2006 from, http://www.cecw-cepb.ca/ DocsEng/DisabilitiesManitobaFinal.pdf

Fuchs, D., Burnside, L., Marchenski, S., \& Mudry, A. (2007). Children with FASD: Involved with the Manitoba Child Welfare System. Retrieved January 11, 2007 from, http://cecw-cepb.ca/files/file/en/ FASD $\% 20$ Final $\% 20$ Report.pdf

Hutson, J. (2006). A prenatal perspective on the cost of substance abuse in Canada. Journal of FAS International, 4(9), $1-4$.

Klug, M. G., \& Burd, L. (2003). Fetal alcohol syndrome prevention: Annual and cumulative cost savings. Neurotoxicology Teratology, 25(6), 763-765.

Lupton, C., Burd, L., \& Harwood, R. (2004). Cost of fetal alcohol spectrum disorders. American Journal of Medical Genetics Part C, $127 C, 42-50$.

McKenzie, B., \& Morrissette, V. (2003). Social work practice with Canadians of Aboriginal background: Guidelines for respectful social work. In A. Al-Krenawi, \& J. R. Graham (Eds.) Multicultural social work in Canada: Working with diverse ethno-racial communities. Don Mills, Ontario: Oxford University Press Canada.

Square, D. (1997). Fetal alcohol syndrome epidemic on Manitoba reserve. Canadian Medical Association Journal, 157(1), 59-60.

Stade, B., Ungar, W. J., Stevens, B., Beyenne, J., \& Koren, G. (2006). The burden of prenatal exposure of alcohol: Measurement of cost. Journal of FAS International, 4(5), 1-14.

Thanh, N. X., \& Jonson, E. (2009). Costs of fetal alcohol spectrum disorder in Alberta, Canada. Canadian Journal of Clinical Pharmacology, 16(1), 80-90.

The Child and Family Services Act. (1985). Government of Manitoba. Retreived July 14, 2008 from, http:// web2.gov.mb.ca/laws/statutes/ccsm/c080e.php

Trocmé, N., Knoke, D., \& Blackstock, C. (2004). Pathways to the overrepresentation of Aboriginal children in Canada's child welfare system. Social Service Review, 75(4), 577-600.

Trocmé, N. M., Tourigny, M., Maclaurin, B., \& Fallon, B. (2005). Major findings from the Canadian incidence study of reported child abuse and neglect. Child Abuse and Neglect, 27(12), 1427-1439.

Williams, R. J., Obaido, F. S., \& McGee, J. M. (1999). Incidence of fetal alcohol syndrome in Northeastern Manitoba. Canadian Journal of Public Health, 90(3), 192-194.

World Health Organization. (2003). Development of the ICF for children and youth-Status report. (WHO/ $H F S / C A S / C / 03.63)$. Geneva: Author. 